\title{
The submillimeter-wave spectrum of the doubly deuterated species of methyl formate $\mathrm{HCOOCD}_{2} \mathrm{H}^{\star}$
}

\author{
L. H. Coudert ${ }^{1}$, L. Margulès ${ }^{2}$, T. R. Huet ${ }^{2}$, R. A. Motiyenko ${ }^{2}$, H. Møllendal ${ }^{3}$, and J.-C. Guillemin ${ }^{4}$ \\ ${ }^{1}$ LISA, UMR 7583 CNRS - Universités Paris Est Créteil et Paris Diderot, 61 avenue du Général de Gaulle, 94010 Créteil, France \\ e-mail: laurent.coudert@lisa.u-pec.fr \\ 2 Laboratoire de Physique des Lasers, Atomes et Molécules, UMR 8523 CNRS - Université Lille I, Bât. P5, \\ 59655 Villeneuve d'Ascq Cedex, France \\ 3 Centre for Theoretical and Computational Chemistry (CTCC), Department of Chemistry, University of Oslo, \\ PO Box 1033 Blindern, 0315 Oslo, Norway \\ ${ }^{4}$ Sciences Chimiques de Rennes, UMR 6226 CNRS - ENSCR, Avenue du Général Leclerc, CS 50837, 35708 Rennes Cedex 7, \\ France
}

Received 27 February 2012 / Accepted 17 April 2012

\section{ABSTRACT}

\begin{abstract}
Context. Astronomical surveys of interstellar molecules, such as those that will be available with the very sensitive ALMA telescope, require preliminary laboratory investigations of the microwave and submillimeter-wave spectra of new molecular species to identify these in the interstellar media.

Aims. We build a linelist that should allow us to detect $\mathrm{HCOOCD}_{2} \mathrm{H}$, provided it is present in the interstellar media in a suitable concentration.

Methods. The experimental spectra of $\mathrm{HCOOCD}_{2} \mathrm{H}$ have been recorded in the microwave and submillimeter-wave energy range. Line frequencies were analyzed using an internal axis method-like treatment taking into account the $\mathrm{CD}_{2} \mathrm{H}$ internal rotation.

Results. 5933 lines of $\mathrm{HCOOCD}_{2} \mathrm{H}$ have been assigned and their frequencies were reproduced with a unitless standard deviation ${ }^{\star \star}$ of 1.6. A linelist with calculated frequencies and intensities was built and spans the $50-660 \mathrm{GHz}$ spectral region. The frequency accuracy is better than $0.1 \mathrm{MHz}$.

Conclusions. The pure rotation spectrum of the $\mathrm{CD}_{2} \mathrm{H}$-methyl formate isotopolog $\left(\mathrm{HCOOCD}_{2} \mathrm{H}\right)$ has been observed in laboratory for the first time.
\end{abstract}

Key words. methods: data analysis - surveys - catalogs - molecular data - techniques: spectroscopic

\section{Introduction}

Deuterated molecules are important for studying and understanding the surface chemistry that takes place in interstellar clouds because they provide us with a tool to measure the $\mathrm{D} / \mathrm{H}$ ratio. For this reason, the abundance of deuterated species have been retrieved for many molecular species. For instance, the mono-deuterated (Saito et al. 2000; Shah \& Wootten 2001), bideuterated (Roueff et al. 2000), and triply deuterated (Lis et al. 2002; van der Tak et al. 2002) species for the ammonia molecule have all been detected and their abundance determined. In this context, a linelist for the doubly deuterated species of methyl formate $\left(\mathrm{HCOOCD}_{2} \mathrm{H}\right)$ is built in this investigation. Because this molecule is non-rigid, it displays a fairly congested spectrum and its transitions should be observed in the extensive line surveys that will be carried out with the ALMA telescope, which will lead to its detection in the interstellar medium (ISM).

Methyl formate $\left(\mathrm{HCOOCH}_{3}\right)$ is a non-rigid complex organic molecule of astrophysical relevance (Nummelin et al. 2000; Koyabashi et al. 2007) that displays a rich microwave spectrum

* Tables 3,6, and 10 are available at the CDS via anonymous ftp to cdsarc.u-strasbg.fr $(130.79 .128 .5)$ or via

http://cdsarc.u-strasbg.fr/viz-bin/qcat?]/A+A/543/A46 $\star \star$ The unitless standard deviation $\chi$ is such that $\chi^{2}=\sum_{i=1}^{N}\left[\left(F_{i}^{\text {obs }}-\right.\right.$ $\left.\left.F_{i}^{\mathrm{cal}}\right) / u_{i}^{\mathrm{obs}}\right]^{2} /(N-P)$ where $F_{i}^{\mathrm{obs}}, F_{i}^{\mathrm{cal}}$, and $u_{i}^{\mathrm{obs}}$ are observed frequencies, calculated frequencies, and experimental uncertainties, respectively, in $\mathrm{MHz} ; N$ is the number of lines; and $P$ is the number of varied parameters. that has been the subject of extensive laboratory studies (Curl Jr 1959; Brown et al. 1975; Bauder 1979; Demaison et al. 1983; Plummer et al. 1984, 1986; Oesterling et al. 1999; Karakawa et al. 2001; Ogata et al. 2004; Carvajal et al. 2007; Ilyushin et al. 2009; Demaison et al. 2010; Tudorie et al. 2011) that led to an accurate determination of its spectroscopic parameters including the height of the potential barrier that hinders the internal rotation of the methyl group.

Many investigations have also been carried out on the rotational spectrum of the isotopic species of methyl formate with a symmetrical $\mathrm{CH}_{3}$ methyl group. The two ${ }^{13} \mathrm{C}$ containing species $\mathrm{H}^{13} \mathrm{COOCH}_{3}$ and $\mathrm{HCOO}^{13} \mathrm{CH}_{3}$ have been analyzed and detected in the ISM by Carvajal et al. (2009, 2010). The microwave spectrum of the deuterium containing species $\mathrm{DCOOCH}_{3}$ has also been observed and analyzed and tentatively detected in Orion (Margulès et al. 2010) and in the protostar IRAS 16293-2422 (Demyk et al. 2010). The two ${ }^{18} \mathrm{O}$ containing species $\mathrm{HC}^{18} \mathrm{OOCH}_{3}$ and $\mathrm{HCO}^{18} \mathrm{OCH}_{3}$ were also analyzed and detected in the ISM by Tercero et al. (2011).

Fewer investigations, however, have been carried out on the isotopic species with an asymmetrical partially deuterated methyl group. The spectrum of the mono-deuterated species $\mathrm{HCOOCH}_{2} \mathrm{D}$ has been recorded and analyzed by Margulès et al. (2009), but this isotopic variant has not yet been detected in the ISM. The microwave spectrum of the doubly deuterated species $\mathrm{HCOOCD}_{2} \mathrm{H}$ has not been studied yet.

Experimental and theoretical investigations of the microwave spectrum of $\mathrm{HCOOCD}_{2} \mathrm{H}$ are undertaken in this paper 
to build a linelist for astrophysical purposes. The microwave spectrum was recorded using Fourier transform, conventional Stark modulation, and submillimeter-wave microwave spectroscopy. Line frequencies were analyzed using the approach developed for the $\mathrm{HCOOCH}_{2} \mathrm{D}$ mono-deuterated species (Margulès et al. 2009). The hyperfine structure of 18 transitions was also analyzed to obtain components of the effective quadrupole coupling tensors of the two deuterium atoms. The linelist spans the 50 to $660 \mathrm{GHz}$ region.

\section{Experimental}

The sample used for the measurements was synthesized as follows: formic acid and sulfuric acid (reagent grade, 95-98\%) were purchased from Aldrich, dideuteromethyl alcohol was purchased from Cambridge Isotope Laboratories, Inc. Formic acid ( $2 \mathrm{~g}, 43 \mathrm{mmol}$ ) and dideuteromethyl alcohol (1.02 g, $30 \mathrm{mmol})$ were introduced in a one-necked cell equipped with a stirring bar and a stopcock. The solution was cooled around $-80{ }^{\circ} \mathrm{C}$ and sulfuric acid $(0.3 \mathrm{~g}, 3 \mathrm{mmol})$ was added. The mixture was then cooled in a liquid nitrogen bath and evacuated in vacuo. The stopcock was closed, the solution was heated up to $40{ }^{\circ} \mathrm{C}$ and stirred overnight at this temperature. The cell was then adapted to a vacuum line equipped with two traps and the solution was distilled. High boiling compounds were trapped in the first trap immersed in a bath cooled at $-70{ }^{\circ} \mathrm{C}$. Dideuterated methyl formate $(1.8 \mathrm{~g}, 29 \mathrm{mmol})$ was condensed in the second trap immersed in a liquid nitrogen bath $\left(-196{ }^{\circ} \mathrm{C}\right)$. The yield of the reaction was $97 \%$ and should only lead to trace amounts of the other doubly deuterated species $\mathrm{DCOOCH}_{2} \mathrm{D}$. Identification by nuclear magnetic resonance (NMR) spectroscopy of $\mathrm{HCOOCD}_{2} \mathrm{H}$ yield: ${ }^{1} \mathrm{H} \mathrm{NMR}\left(\mathrm{CDCl}_{3}, 400 \mathrm{MHz}\right)$ 3.73 (d.quint, ${ }^{1} \mathrm{H},{ }^{4} J_{\mathrm{HH}}=0.7 \mathrm{~Hz},{ }^{2} J_{\mathrm{HD}}=0.9 \mathrm{~Hz}, \mathrm{CHD}_{2}$ ); $8.08\left(\mathrm{~d},{ }^{1} \mathrm{H},{ }^{4} \mathrm{~J}_{\mathrm{HH}}=0.7 \mathrm{~Hz}, \mathrm{CHO}\right) .{ }^{13} \mathrm{C} \mathrm{NMR}\left(\mathrm{CDCl}_{3}, 100 \mathrm{MHz}\right)$ $50.2\left({ }^{1} J_{\mathrm{CD}}=22.5 \mathrm{~Hz}\right.$ (quint) $) ; 161.2(\mathrm{CHO})$. Three sets of measurements were carried out with this sample.

Set A was performed using the new molecular beam Fourier transform microwave spectrometer in Lille that spans the 2-20 GHz spectral range (Tudorie et al. 2011). Methyl formate vapors at a pressure of 30 mbar were mixed with neon carrier gas at a backing pressure of 1.3 bar, and a frequency domain signal was obtained just as in Tudorie et al. (2011). With this spectrometer, which allows us to reach a temperature as low as $2 \mathrm{~K}$, each molecular line gives rise to a Doppler doublet. A total of 28 transitions were recorded and we were able to resolve the tunneling splitting due to a tunneling motion in the lowest lying configuration, as described in Sect. 3.2. For 18 lines, the quadrupole coupling hyperfine structure was also resolved. A typical example of the signal associated with the non-tunneling component of the $J_{K_{a} K_{c}}=1_{01} \leftarrow 0_{00}$ rotational transitions is displayed in Fig. 1, where a calculated hyperfine pattern is also shown. The analysis of the hyperfine patterns, described in Sect. 3.4, provided us with useful information about the conformation structures and with 18 accurate center frequencies.

Set B was carried out in the $7-80 \mathrm{GHz}$ region using the Stark-modulated spectrometer of the University of Oslo. Details of the construction and operation of this spectrometer have been given elsewhere (Møllendal et al. 2005, 2006). The spectrum was recorded at room temperature, or at roughly $-20^{\circ} \mathrm{C}$, at a pressure of approximately $10 \mathrm{~Pa}$, employing a Stark field strength of about $1100 \mathrm{~V} / \mathrm{cm}$. The frequency of 87 individual transitions was measured with an estimated accuracy of $0.1 \mathrm{MHz}$.
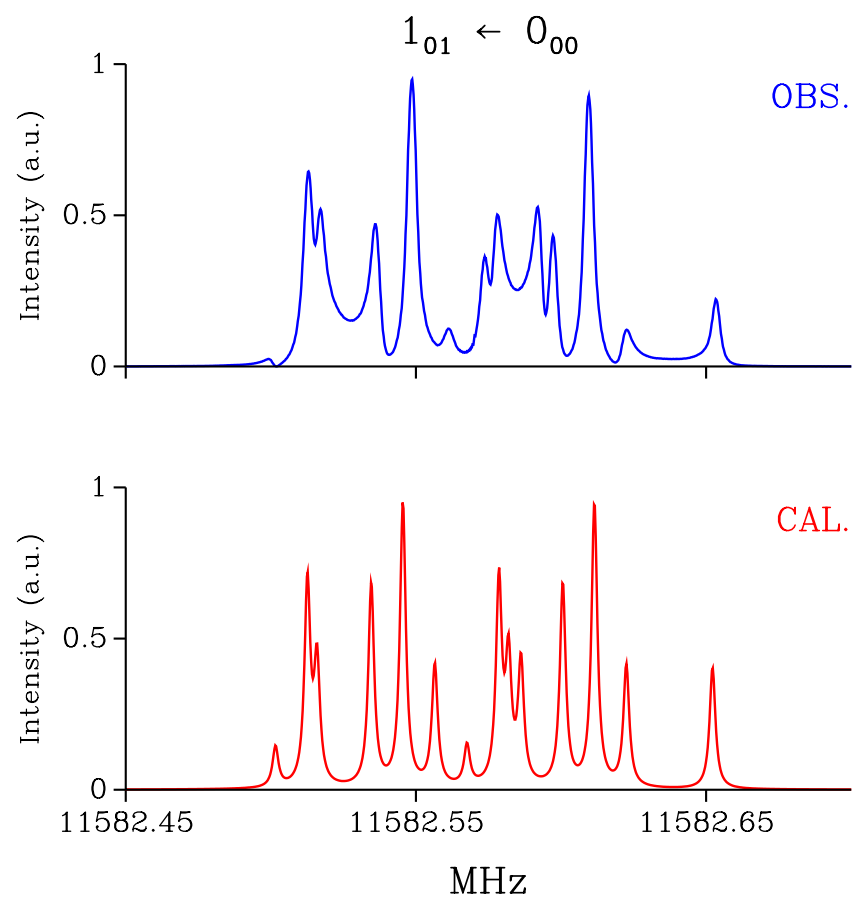

Fig. 1. Non-tunneling component of the $1_{01} \leftarrow 0_{00}$ rotational transition of doubly deuterated methyl formate recorded with the Fourier transform microwave spectrometer of Set A measurements. The quadrupole hyperfine structure associated with the two deuterium atoms can clearly be seen in the observed spectrum displayed in the upper part of the figure. The lower part of the figure shows the calculated spectrum. A Lorentzian lineshape with a half width at half height of $1.2 \mathrm{kHz}$ was assumed for each hyperfine component. The experimental Doppler splitting due to the molecular beam spectrometer was taken to be equal to $66 \mathrm{kHz}$. The labeling of the transitions is presented in Sect. 3.1 and the hyperfine patterns are analyzed in Sect. 3.4.

The submillimeter-wave measurements of Set $\mathrm{C}$ were performed from 150 to $660 \mathrm{GHz}$ using the Lille spectrometer (Motiyenko et al. 2010). In the frequency ranges 150-322 and $400-533 \mathrm{GHz}$, a solid state frequency multiplication chain was used as source of radiation. The spectra in the 580 to $660 \mathrm{GHz}$ frequency range were recorded using our new fast-scan spectrometer (Alekseev et al. 2012). It is based on an Istok backward wave oscillator (BWO) locked to a harmonic of the Agilent synthesizer E8257D (8-18 GHz). A high-resolution fast frequency scan (up to $100 \mathrm{GHz} / \mathrm{h}$ ) was obtained by a direct digital synthesizer used as a reference source of BWO's phase locked loop (PLL). As a detector we used an InSb liquid He-cooled bolometer from QMC instruments Ltd. The absorption cell was a stainless steel tube (6 cm diameter, $220 \mathrm{~cm}$ long). The sample pressure during measurements was about $2.5 \mathrm{~Pa}$ and the linewidth was limited by Doppler broadening. The measurement accuracy for an isolated line is estimated to be better than $30 \mathrm{kHz}$. However if the lines were blended or with a poor signal-to-noise ratio, an experimental uncertainty of 100 or even $200 \mathrm{kHz}$ was assumed. A typical trace obtained with this experimental setup is shown in Fig. 2.

\section{Assignment and analysis of the spectrum}

\subsection{Theoretical model}

The rotation-torsion energy levels of doubly deuterated methyl formate were calculated using the model developed for the mono-deuterated species (Margulès et al. 2009). Owing to 


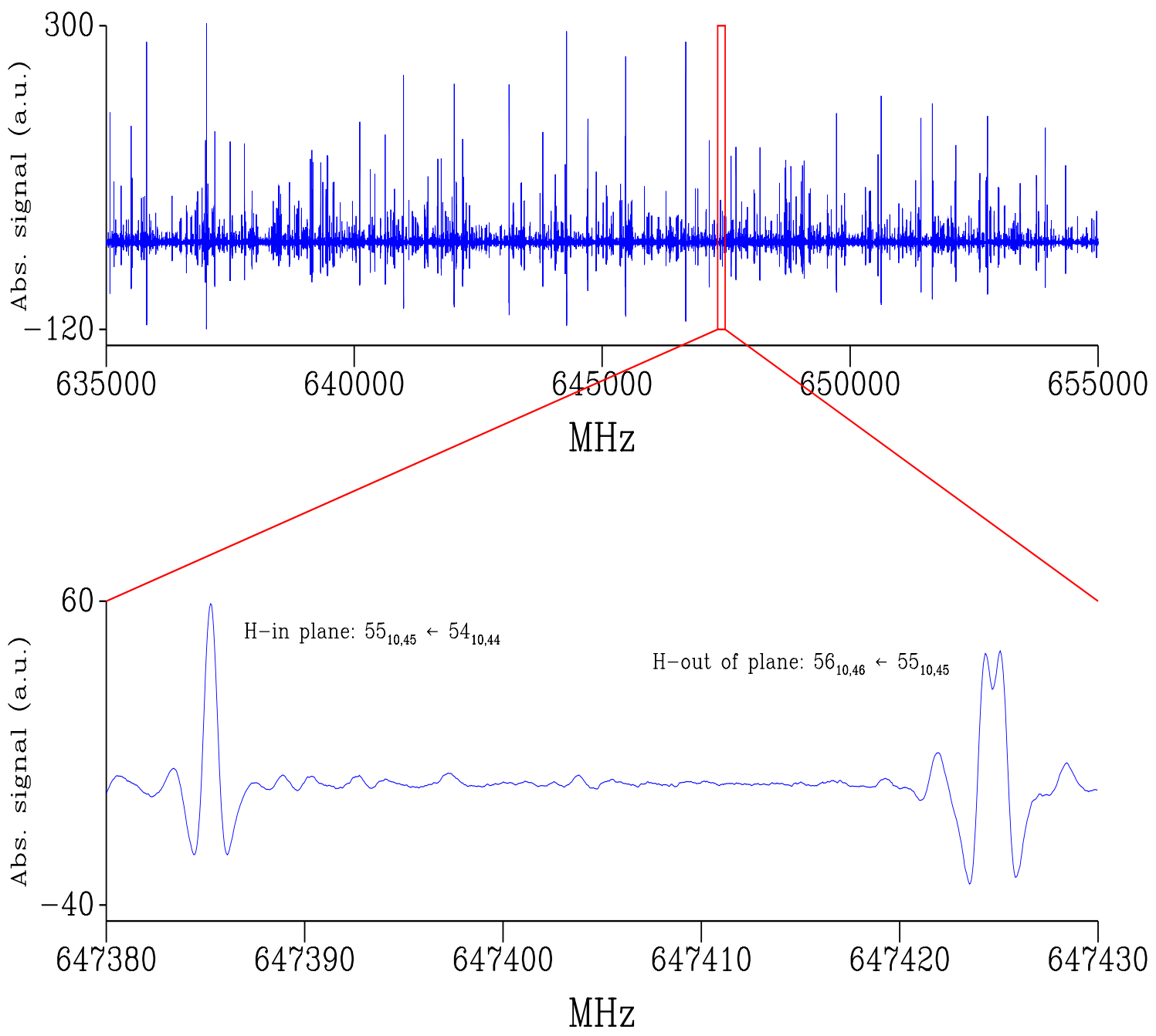

Fig. 2. Two portions of the submillimeter-wave spectrum recorded with the Set $\mathrm{C}$ experimental setup are shown. Both traces are the second derivative of the actual spectrum profile due to signal demodulation at $2 F$. The $x$-axis is the frequency in $\mathrm{MHz}$, the $y$-axis is the absorption signal in arbitrary units. The upper part of the figure shows the portion from 635 to $655 \mathrm{GHz}$ where $R$-type lines can be seen that are likely candidates for an astrophysical detection. The lower part shows the smaller portion of the spectrum from 647380 to $647430 \mathrm{MHz}$ where two transitions are labeled. The tunneling splitting can be seen for the transition corresponding to the H-out-of-plane configurations.

zero-point energy effects, the energy level diagram of $\mathrm{HCOOCD} 2 \mathrm{H}$ is qualitatively different from that of $\mathrm{HCOOCH}_{2} \mathrm{D}$, as shown in Fig. 3. In $\mathrm{HCOOCD}_{2} \mathrm{H}$, the isolated sublevel of $A^{\prime}$ symmetry has the highest energy. This sublevel corresponds to the so-called $\mathrm{H}$-in-plane configuration such that the only hydrogen atom of the methyl group lies in the molecular symmetry plane. The rotational levels arising from this configuration can be calculated using a Watson-type Hamiltonian. The two close-lying sublevels, of symmetry $A^{\prime}$ and $A^{\prime \prime}$, below the isolated sublevel, are tunneling sublevels arising from hindered rotation of the methyl group. The torsional motion can connect the two so-called H-out-of-plane configurations with the only hydrogen atom of the methyl group above or below the plane that contains the HCOO atoms. The internal axis method (IAM) approach (Hougen 1985; Coudert \& Hougen 1988) developed for the mono-deuterated species (Margulès et al. 2009) allows us to calculate the rotational energies arising from these two configurations because it accounts for the rotational dependence of the tunneling splitting. Rotational levels arising from the $\mathrm{H}$-in-plane configuration are labeled using the usual asymmetric top rotational quantum numbers $J, K_{a}$, and $K_{c}$. Rotational levels corresponding to the two H-out-of-plane configurations will be labeled using these rotational quantum numbers and the + or - sign depending on whether the level arises from the lower or upper tunneling sublevel, respectively.

\subsection{Transitions assignment}

The Set A centimeter-wave spectrum was assigned first. Strong, low $J, a$-type transitions were identified starting from spectroscopic constants calculated from the structure. Using a bootstrap approach, a preliminary line frequency analysis was carried out and new transitions were predicted and searched for. This procedure was repeated and allowed us to also assign $a$-type transitions in Sets B and C. When enough of those were available, $b$-type transitions were also assigned for all measurement sets. For the H-out-of-plane configurations, a few $c$-type transitions could be assigned. Table 1 gives the number of assigned transitions for configuration along with maximum values of $J$ and $K_{a}$.

\subsection{Line frequency analysis}

The microwave data recorded in our investigation were analyzed calculating the tunneling-rotational energy with the theoretical approach developed for the mono-deuterated species 


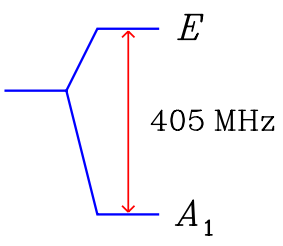

$\mathrm{HCOOCH}_{3}$

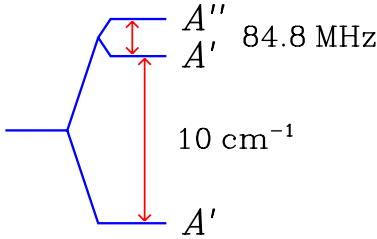

$\mathrm{HCOOCH}_{2} \mathrm{D}$

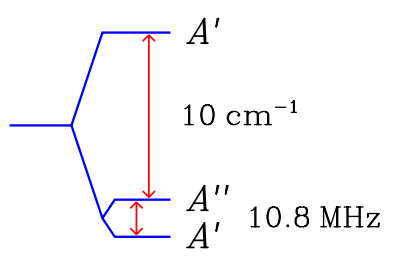

$\mathrm{HCOOCD}_{2} \mathrm{H}$
Fig. 3. Torsional energy pattern of $\mathrm{HCOOCH}_{3}, \mathrm{HCOOCH}_{2} \mathrm{D}$, and $\mathrm{HCOOCD}_{2} \mathrm{H}$. The energy pattern consists of two sublevels, $405 \mathrm{MHz}$ apart, for the lighter symmetrical species (Ilyushin et al. 2009) which belong to the $A_{1}$ and $E$ symmetry species of $C_{3 v}$. For the monodeuterated species, three nondegenerate sublevels arise. Two closelying $A^{\prime}$ and $A^{\prime \prime}$ levels, only $80 \mathrm{MHz}$ apart, can be seen about $10 \mathrm{~cm}^{-1}$ above an $A^{\prime}$ isolated sublevel (Margulès et al. 2009). For the doubly deuterated species, the pair of close-lying levels is about $10 \mathrm{~cm}^{-1}$ below the isolated sublevel. For this species, the tunneling splitting is $10.8 \mathrm{MHz}$ as obtained in this work.

Table 1. Number of assigned transitions for the various configurations.

\begin{tabular}{lcccccc}
\hline \hline Configuration & $N$ & $a$-type & $b$-type & $c$-type & $J(\operatorname{Max})$ & $K_{a}(\operatorname{Max})$ \\
\hline H-in-plane & 2295 & 1191 & 1104 & 0 & 60 & 37 \\
H-out-of-plane & 3638 & 1956 & 1565 & 65 & 60 & 40 \\
\hline Both & 5933 & 3147 & 2669 & 65 & 60 & 40 \\
\hline
\end{tabular}

(Margulès et al. 2009). Experimental frequencies were introduced in a least-squares fit procedure where they were given a weight equal to the inverse of the square of their experimental uncertainty. Unresolved doublets were treated as follows: assuming that such a doublet corresponds to the two transitions $J^{\prime} K_{a 1}^{\prime} K_{c 1}^{\prime}, \pm \leftarrow J^{\prime \prime} K_{a 1}^{\prime \prime} K_{c 1}^{\prime \prime}, \pm$ and $J^{\prime} K_{a 2}^{\prime} K_{c 2}^{\prime}, \pm \leftarrow J^{\prime \prime} K_{a 2}^{\prime \prime} K_{c 2}^{\prime \prime}, \pm$ with calculated frequencies $F_{1}$ and $F_{2}$, respectively. The doublet was treated in the analysis as a single data point with a calculated frequency equal to $\left(F_{1}+F_{2}\right) / 2$. In the present data set, usual unresolved $K$-type doublets arise for all configurations. For the $\mathrm{H}$-out-of-plane configurations, doublets also arise because of unresolved tunneling components. Quartets are also observed and are due to unresolved $K$-type and tunneling components.

For the 5933 fitted transitions, the root-mean-square (rms) value of the observed minus calculated residual is $0.047 \mathrm{MHz}$ and the unitless standard deviation is 1.6. For the 2295 (3638) transitions corresponding to the $\mathrm{H}$-in-plane configuration (H-out-of-plane configurations) only, the rms value is $0.039 \mathrm{MHz}(0.051 \mathrm{MHz})$. Table 2 lists assignments, observed frequencies, and observed minus calculated differences for the 40 lowest frequency transitions. Table 3, available at the CDS, lists the same quantities for all transitions. These quantities are displayed using 12 columns. Columns 1 to 4 (5 to 8 ) give the assignment of the upper (lower) level in terms of $J, K_{a}, K_{c}$, and the configuration label. The latter is blank for the H-in-plane configuration and + or - for the H-out-of-plane configurations (see Sect. 3.1). Column 9 is the observed frequency in $\mathrm{MHz}$ with its uncertainty given in parentheses in $\mathrm{kHz}$; Col. 10 is the observed minus calculated residual in $\mathrm{kHz}$; Col. 11 indicates whether the line belongs to a doublet or to a quartet, and Col. 12 describes the measurement set from which the transition was taken using the labeling of Sect. 2. Tables 4 and 5 give the values and the uncertainties of the parameters determined in the analysis for the $\mathrm{H}$-in-plane and $\mathrm{H}$-out-of-plane configurations, respectively.

\subsection{Hyperfine structure analysis}

The hyperfine splittings observed for several transitions in Set A are caused by quadrupole coupling arising from both deuterium atoms. Numbering these two atoms 2 and 3 as in Margulès et al. (2009), their nuclear momentum will be $\boldsymbol{I}_{2}$ and $\boldsymbol{I}_{3}$, respectively. The matrix of the effective quadrupole coupling Hamiltonian, as given in Eq. (1) of Coudert et al. (1989), is set-up using the $\left|\left(I_{2}, I_{3}\right) I J F M_{F}\right\rangle$ basis set that corresponds to the coupling scheme $\boldsymbol{I}=\boldsymbol{I}_{2}+\boldsymbol{I}_{3}$ and $\boldsymbol{F}=\boldsymbol{I}+\boldsymbol{J}$. Since $\boldsymbol{I}_{2}=I_{3}=1$, the quantum number for the total nuclear spin angular momentum $I$ is 0,1 , or 2; and the quantum number for the total angular momentum $F$ takes the five values $J-2, J-1, J, J+1$, and $J+2$, for $J \geq 2$. For $J=1, F$ only takes the four values $0,1,2$, and 3 ; and for $J=0, F$ only takes the three values 0,1 , and 2 . Matrix elements of the effective quadrupole coupling Hamiltonian with this coupling scheme were derived by Robinson \& Cornwell (1953). These results show that nine hyperfine levels arise for $J \geq 2$, seven for $J=1$, and only three for $J=0$.

The hyperfine patterns were analyzed using a least-squares fit procedure in which the hyperfine energy is computed as outlined in the previous paragraph. For the H-in-plane configuration, the following components of the effective quadrupole coupling tensors were determined:

$e Q q_{x x}^{2}=e Q q_{x x}^{3}, e Q q_{y y}^{2}=e Q q_{y y}^{3}$,

where superscripts 2 and 3 identify the two deuterium atoms. For the H-out-of-plane configurations, only the following linear combinations of effective quadrupole coupling tensor components could be determined because of the tunneling averaging:

$\left(e Q q_{x x}^{2}+e Q q_{x x}^{3}\right) / 2, \quad\left(e Q q_{y y}^{2}+e Q q_{y y}^{3}\right) / 2$,

where superscripts 2 and 3 have the same meaning as in Eq. (1). Table 6, available at the CDS and displaying 12 columns, gives the transition assignment (Cols. 1 to 8 ), the center frequency (Col. 9), its uncertainty in $\mathrm{kHz}$ (Col. 10), the number of hyperfine components (Col. 11), and the rms deviation (Col. 12) for the fitted hyperfine patterns. The center frequencies can also be found in Table 2. Fitted components of the effective quadrupole coupling tensors are listed in Table 7 where they are compared to values obtained through ab initio calculations (Demaison 2009, priv. comm.; Demaison et al. 2010).

\section{Intensity calculation}

Line strengths were calculated using the values for the dipole moment components listed in Table 8, retrieved from those of the normal species. The values reported for the latter species by Curl Jr (1959) were $\mu_{a}=1.63, \mu_{b}=0.68$, and $\mu_{\mathrm{tot}}=1.77 \mathrm{D}$, without quoted uncertainties. Measurements carried out recently by Margulès et al. (2010) led to the more accurate values $\mu_{a}=$ $1.648(8), \mu_{b}=0.706(12)$, and $\mu_{\mathrm{tot}}=1.793(11) \mathrm{D}$. The partition 


\section{H. Coudert et al.: Microwave spectrum of $\mathrm{HCOOCD}_{2} \mathrm{H}$}

Table 2. Assignments, observed frequencies, and observed minus calculated differences in the microwave spectrum of $\mathrm{HCOOCD}_{2} \mathrm{H}$.

\begin{tabular}{|c|c|c|c|c|c|c|c|c|c|c|c|c|c|c|c|c|c|c|c|c|c|}
\hline$J^{\prime}$ & $K_{a}^{\prime}$ & $K_{c}^{\prime}$ & \pm & $J^{\prime \prime}$ & $K_{a}^{\prime \prime}$ & $K_{c}^{\prime \prime}$ & \pm & $\mathrm{Obs}^{a}$ & $\operatorname{Diff}^{c}$ & $\mathrm{Set}^{d}$ & $J^{\prime}$ & $K_{a}^{\prime}$ & $K_{c}^{\prime}$ & \pm & $J^{\prime \prime}$ & $K_{a}^{\prime \prime}$ & $K_{c}^{\prime \prime}$ & \pm & $\mathrm{Obs}^{a}$ & $\operatorname{Diff}^{c}$ & $\operatorname{Set}^{d}$ \\
\hline 2 & 1 & 1 & + & 2 & 1 & 2 & + & $4130.528(30)$ & -3 & $\mathrm{~A}$ & 4 & 1 & 3 & - & 4 & 1 & 4 & - & $13728.493(2)^{b}$ & 3 & $\mathrm{~A}$ \\
\hline 6 & 2 & 4 & + & 6 & 2 & 5 & + & $6861.507(30)$ & 8 & A & 4 & 1 & 3 & + & 4 & 1 & 4 & + & $13729.199(2)^{b}$ & 12 & A \\
\hline 3 & 1 & 2 & - & 3 & 1 & 3 & - & $8254.912(30)$ & 0 & A & 2 & 1 & 1 & & 2 & 0 & 2 & & $13884.674(1)^{b}$ & 1 & $\mathrm{~A}$ \\
\hline 3 & 1 & 2 & + & 3 & 1 & 3 & + & $8255.339(30)$ & 5 & A & 18 & 5 & 13 & & 18 & 5 & 14 & & $14882.480(150)$ & -171 & $\mathrm{~B}$ \\
\hline 2 & 0 & 2 & + & 1 & 1 & 1 & + & $9959.041(1)^{b}$ & -1 & A & 4 & 1 & 3 & & 4 & 1 & 4 & & $14922.583(1)^{b}$ & -1 & A \\
\hline 2 & 0 & 2 & - & 1 & 1 & 1 & - & $9959.310(1)^{b}$ & 1 & A & 2 & 1 & 1 & - & 2 & 0 & 2 & - & $15084.586(1)^{b}$ & 0 & $\mathrm{~A}$ \\
\hline 1 & 0 & 1 & - & 0 & 0 & 0 & - & $11145.210(1)^{b}$ & 10 & A & 2 & 1 & 1 & + & 2 & 0 & 2 & + & $15085.130(1)^{b}$ & 6 & A \\
\hline 1 & 0 & 1 & + & 0 & 0 & 0 & + & $11145.267(1)^{b}$ & -1 & A & 34 & 9 & 25 & & 34 & 9 & 26 & & $15319.840(150)$ & -331 & B \\
\hline 7 & 1 & 7 & + & 6 & 2 & 4 & + & $11401.310(30)$ & -31 & A & 22 & 6 & 16 & & 22 & 6 & 17 & & $15589.120(150)-$ & -107 & B \\
\hline 7 & 1 & 7 & - & 6 & 2 & 4 & - & $11403.493(30)$ & -4 & A & 26 & 7 & 19 & & 26 & 7 & 20 & & $15840.000(150)$ & 3 & B \\
\hline 7 & 2 & 5 & - & 7 & 2 & 6 & - & $11514.713(30)$ & 7 & A & 3 & 1 & 2 & & 3 & 0 & 3 & & $16611.700(1)^{b}$ & -2 & A \\
\hline 7 & 2 & 5 & + & 7 & 2 & 6 & + & $11515.400(30)$ & 15 & A & 3 & 1 & 2 & - & 3 & 0 & 3 & - & $17513.025(3)^{b}$ & 0 & A \\
\hline 1 & 0 & 1 & & 0 & 0 & 0 & & $11582.571(1)^{b}$ & -1 & A & 3 & 1 & 2 & + & 3 & 0 & 3 & + & $17513.691(3)^{b}$ & 7 & $\mathrm{~A}$ \\
\hline 1 & 1 & 0 & & 1 & 0 & 1 & & $12239.931(1)^{b}$ & -6 & A & 11 & 3 & 8 & & 11 & 3 & 9 & & $18090.260(150)$ & 22 & $\mathrm{~B}$ \\
\hline 2 & 0 & 2 & & 1 & 1 & 1 & & $12277.561(1)^{b}$ & -4 & A & 15 & 4 & 11 & & 15 & 4 & 12 & & $20664.460(150)$ & -294 & $\mathrm{~B}$ \\
\hline 4 & 1 & 3 & + & 3 & 2 & 2 & + & $12425.644(30)$ & 2 & A & 4 & 1 & 3 & & 4 & 0 & 4 & & $20672.400(150)$ & 127 & B \\
\hline 4 & 1 & 3 & - & 3 & 2 & 2 & - & $12426.284(30)$ & 3 & A & 2 & 1 & 2 & & 1 & 1 & 1 & & $21666.090(150)-$ & -367 & B \\
\hline 1 & 1 & 0 & - & 1 & 0 & 1 & - & $13597.872(1)^{b}$ & -9 & A & 19 & 5 & 14 & & 19 & 5 & 15 & & $22323.440(150)$ & 328 & B \\
\hline 1 & 1 & 0 & + & 1 & 0 & 1 & + & $13598.344(1)^{b}$ & -4 & A & 2 & 0 & 2 & & 1 & 0 & 1 & & $23018.640(150)$ & -240 & B \\
\hline 14 & 4 & 10 & & 14 & 4 & 11 & & $13609.710(150)$ & -47 & B & 23 & 6 & 17 & & 23 & 6 & 18 & & $23236.730(150)-$ & -219 & B \\
\hline
\end{tabular}

Notes. Transitions are assigned with the usual rotational quantum numbers of the upper and lower levels. For transitions within the H-out-of-plane configurations, the + or - signs identify tunneling sublevels. Only the 40 lowest frequency transitions appear in this table. ${ }^{(a)}$ Obs is the observed frequency in MHz. The uncertainty is given in parentheses in kHz. ${ }^{(b)}$ Obtained in the hyperfine structure analysis of Sect. 3.4. ${ }^{(c)}$ Diff is the observed minus calculated difference in kHz. ${ }^{(d)}$ The measurement set as defined in Sect. 2 is given.

Table 4. Spectroscopic parameters for the H-in-plane configuration.

\begin{tabular}{lccc}
\hline \hline Parameter & Value & Parameter & Value \\
\hline$A$ & $17281.949265(420)$ & $H_{K J J} \times 10^{9}$ & $-210.249(540)$ \\
$B$ & $6540.604314(110)$ & $H_{J J J} \times 10^{9}$ & $2.332(19)$ \\
$C$ & $5041.990952(120)$ & $h_{K K} \times 10^{9}$ & $-601.454(3897)$ \\
& & $h_{K J} \times 10^{9}$ & $330.458(839)$ \\
$\Delta_{K K} \times 10^{3}$ & $41.404648(2200)$ & $h_{J J} \times 10^{9}$ & $1.322(7)$ \\
$\Delta_{K J} \times 10^{3}$ & $-7.932466(660)$ & & \\
$\Delta_{J J} \times 10^{3}$ & $5.806476(90)$ & $L_{K K K K} \times 10^{12}$ & $-14.173(2998)$ \\
$\delta_{K} \times 10^{3}$ & $-5.786705(1000)$ & $L_{K K K J} \times 10^{12}$ & $4.787(720)$ \\
$\delta_{J} \times 10^{3}$ & $1.787297(26)$ & $L_{K J J J} \times 10^{12}$ & $-4.897(72)$ \\
& & $l_{K K J} \times 10^{12}$ & $4.777(839)$ \\
$H_{K K K} \times 10^{9}$ & $123.268(4797)$ & $l_{K J J} \times 10^{12}$ & $7.766(162)$ \\
$H_{K K J} \times 10^{9}$ & $611.220(1469)$ & & \\
\hline
\end{tabular}

Notes. Parameters are in MHz. Numbers in parentheses are one standard deviation in the same units as the last digit. These parameters are involved in the pure rotational Hamiltonian in the second of Eqs. (10) of Margulès et al. (2009).

function $Q_{\text {rot }}$ was computed for several temperatures taking a degeneracy factors equal to $(2 J+1)$. The energy of the $0_{00},+$ level corresponding to the H-out-of-plane configurations was taken to be equal to zero, that of the $0_{00}$ level of the $\mathrm{H}$-in-plane configuration was set to $10 \mathrm{~cm}^{-1}$ as in Sect. 3.1. Table 9 gives the values thus obtained for the partition functions.

The linelist was built calculating the frequencies of the allowed transitions characterized by a lower and an upper rotational quantum number $J$ smaller than 60 . Integrated intensities were calculated in $\mathrm{nm}^{2} \mathrm{MHz}$ units at $300 \mathrm{~K}$ using the results given in Tables 8 and 9. Just as in the JPL (Pickett et al. 1998) data bases, the selected transitions are those with an intensity in $\mathrm{nm}^{2} \mathrm{MHz}$ units at $300 \mathrm{~K}$ larger than

$10^{\text {LOGSTR0 }}+(F / 300000)^{2} \times 10^{\text {LOGSTR1 }}$,
Table 5. Spectroscopic parameters for the H-out-of-plane configurations.

\begin{tabular}{lccc}
\hline \hline Parameter & Value & Parameter & Value \\
\hline$\theta_{2}$ & $6.655648(2600)$ & $H_{K K K} \times 10^{9}$ & $1195.553(5096)$ \\
$\phi_{2}$ & $81.578313(5700)$ & $H_{K K J} \times 10^{9}$ & $-461.461(1289)$ \\
& & $H_{K J J} \times 10^{9}$ & $-34.508(420)$ \\
$h_{2}$ & $-5.394756(8600)$ & $H_{J J J} \times 10^{9}$ & $-1.833(54)$ \\
$h_{2 k} \times 10^{6}$ & $2914.209(47967)$ & $h_{K K} \times 10^{9}$ & $357.691(5396)$ \\
$h_{2 j} \times 10^{6}$ & $-1184.396(10193)$ & $h_{K J} \times 10^{9}$ & $101.726(510)$ \\
$f_{2} \times 10^{6}$ & $-213.732(4497)$ & $h_{J J} \times 10^{9}$ & $-0.878(5)$ \\
$A$ & $18482.353793(330)$ & $L_{K K K K} \times 10^{12}$ & $-114.585(14390)$ \\
$B$ & $6261.052643(100)$ & $L_{K K K J} \times 10^{12}$ & $159.564(21585)$ \\
$C$ & $4884.201398(110)$ & $L_{K K J J} \times 10^{12}$ & $-55.071(7495)$ \\
& & $L_{K J J J} \times 10^{12}$ & $-5.095(48)$ \\
$\Delta_{K K} \times 10^{3}$ & $57.573479(2000)$ & $L_{J J J J} \times 10^{12}$ & $-0.058(8)$ \\
$\Delta_{K J} \times 10^{3}$ & $-13.498154(360)$ & $l_{K K K} \times 10^{12}$ & $-308.607(59958)$ \\
$\Delta_{J J} \times 10^{3}$ & $5.036334(120)$ & & \\
$\delta_{K} \times 10^{3}$ & $1.622568(630)$ & & \\
$\delta_{J} \times 10^{3}$ & $1.540159(21)$ & & \\
\hline
\end{tabular}

Notes. Parameters are in $\mathrm{MHz}$ except for $\theta_{2}$ and $\phi_{2}$, which are in degrees. Numbers in parentheses are one standard deviation in the same units as the last digit. Parameters are defined in Eqs. (12), (13), and (22) of Margulès et al. (2009) or are involved in the pure rotational Hamiltonian in the first of Eqs. (10) of the same reference.

where $F$ is the frequency in MHz, and LOGSTR0 and LOGSTR1 are two dimensionless constants set to -9 and -7 , respectively. The uncertainty on the calculated frequency was retrieved from the analysis results.

The linelist is given in Table 10, available at the CDS. It is formatted in the same way as the catalog line files of the JPL data base (Pickett et al. 1998) and has 16 columns. The first, second, and third columns contain the line frequency (FREQ) in $\mathrm{MHz}$, the error (ERR) in $\mathrm{MHz}$, and the base 10 logarithm of the line 
Table 7. Effective quadrupole coupling tensors components in MHz.

\begin{tabular}{lrr}
\hline \hline Component & \multicolumn{1}{c}{ Value $^{a}$} & \multicolumn{1}{c}{ Ab initio $^{b}$} \\
\hline$e Q q_{x x}^{2}=e Q q_{x x}^{3}$ & $-0.0087(14)$ & -0.0071 \\
$e Q q_{y y}^{2}=e Q q_{y y}^{3}$ & $0.0819(17)$ & 0.0935 \\
$\left(e Q q_{x x}^{2}+e Q q_{x x}^{3}\right) / 2$ & $-0.0311(10)$ & -0.0305 \\
$\left(e Q q_{y y}^{3}+e Q q_{y y}^{3}\right) / 2$ & $0.0063(14)$ & -0.0106 \\
\hline
\end{tabular}

Notes. Superscripts 2 and 3 identify the deuterium atoms. The two first (last) lines correspond to the H-in-plane configuration (H-out-of-plane configurations). ${ }^{(a)}$ Fitted values obtained in Sect. 3.4 are reported. Numbers in parentheses are one standard deviation in the same units as the last digit. ${ }^{(b)}$ From Demaison (2009, priv. comm.), Demaison et al. (2010).

Table 8. Numerical values, in Debye, used for the dipole moment components matrix elements in the line intensity calculation.

\begin{tabular}{lclc}
\hline \hline Configuration & $\mu_{x}$ & $\mu_{y}$ & $\mu_{z}$ \\
\hline H-in-plane & 0.792 & 0.0 & 1.608 \\
H-out-of-plane & 0.763 & 0.011 & 1.622 \\
\hline
\end{tabular}

Notes. Dipole moment components were retrieved from those of the normal species (Margulès et al. 2010).

Table 9. Partition function $Q_{\text {rot }}$ of $\mathrm{HCOOCD}_{2} \mathrm{H}$ for several values of the temperature $T$ in Kelvin.

\begin{tabular}{rrrr}
\hline \hline$T / \mathrm{K}$ & \multicolumn{1}{c}{$Q_{\text {rot }}$} & \multicolumn{1}{c}{$T / \mathrm{K}$} & \multicolumn{1}{c}{$Q_{\text {rot }}$} \\
\hline 9.375 & 455 & 150.000 & 37839 \\
18.750 & 1424 & 225.000 & 69294 \\
37.500 & 4372 & 300.000 & 104036 \\
75.000 & 13020 & & \\
\hline
\end{tabular}

Notes. The energy of the $J=0, A^{\prime}$ sublevel of the H-out-of-plane configurations was taken to be equal to zero; that of the $J=0, A^{\prime}$ level of the $\mathrm{H}$-in-plane configuration was taken to be equal to $10 \mathrm{~cm}^{-1}$ (see Fig. 3).

intensity (LGINT) in $\mathrm{nm}^{2} \mathrm{MHz}$ at $300 \mathrm{~K}$. The fourth, fifth, and sixth columns give the degrees of freedom of the rotational partition function (DR), the lower state energy (ELO) in $\mathrm{cm}^{-1}$, and the upper state degeneracy (GUP), respectively. The seventh and eighth columns contain the species tag (TAG) and format number (QNFMT), respectively. Finally, Cols. 9 to 12 (13 to 16) give the assignment of the upper (lower) level in terms of $J, K_{a}, K_{c}$, and the configuration label. The latter is 0 or 1 for the + and - levels of the H-out-of-plane configurations and 2 for the H-in-plane configuration. When the calculated error (ERR) was smaller than $10 \mathrm{kHz}$, it was set to that value. For observed unblended microwave lines, the line frequency (FREQ) and the error (ERR) were replaced by their experimental values. This is then indicated by a negative species tag (TAG).

\section{Conclusion}

The pure rotation spectrum of $\mathrm{HCOOCD}_{2} \mathrm{H}$ was observed in laboratory up to $660 \mathrm{GHz}$. Almost six thousand lines were assigned to the $\mathrm{H}$-in-plane and $\mathrm{H}$-out-of-plane configurations. The large amplitude torsional motion of the partially deuterated $\mathrm{CD}_{2} \mathrm{H}$ methyl group was accounted for in the energy level calculation. The spectroscopic constants given in Tables 4 and 5 were determined and allowed us to reproduce the observed frequencies with an accuracy better than $100 \mathrm{kHz}$. The linelist built using the results of this analysis should allow the detection of $\mathrm{HCOOCD}_{2} \mathrm{H}$, provided it is present in the ISM in a suitable concentration.

Acknowledgements. This work was supported by the French program Action sur Projets de 1'INSU "Physique et Chimie du Milieu Interstellaire" and by the contracts ANR-08-BLAN-0054 and ANR-08-BLAN-0225.

\section{References}

Alekseev, E. A., Motiyenko, R. A., \& Margulès, L. 2012, Radio Phys. Radio Astron., 3, 75

Bauder, A. 1979, J. Phys. Chem. Ref. Data, 8, 583

Brown, R. D., Crofts, J. G., Gardner, F. F., et al. 1975, ApJ, 197, L29

Carvajal, M., Willaert, F., Demaison, J., \& Kleiner, I. 2007, J. Mol. Spectrosc., 246,158

Carvajal, M., Margulès, L., Tercero, B., et al. 2009, A\&A, 500, 1109

Carvajal, M., Kleiner, I., \& Demaison, J. 2010, ApJS, 190, 315

Coudert, L. H., \& Hougen, J. T. 1988, J. Mol. Spectrosc., 130, 86

Coudert, L. H., Hougen, J. T., \& Suenram, R. D. 1989, J. Mol. Spectrosc., 135, 314

Curl, Jr, R. F. 1959, J. Chem. Phys., 30, 1529

Demaison, J., Boucher, D., Dubrulle, A., \& van Eijck, B. P. 1983, J. Mol. Spectrosc., 102, 260

Demaison, J., Margulès, L., Kleiner, I., \& Császár, A. G. 2010, J. Mol Spectrosc., 259, 70

Demyk, K., Bottinelli, S., Caux, E., et al. 2010, A\&A, 517, A17

Hougen, J. T. 1985, J. Mol. Spectrosc., 114, 395

Ilyushin, V., Kryvda, A., \& Alekseev, E. 2009, J. Mol. Spectrosc., 255, 32

Karakawa, Y., Oka, K., Odashima, H., Takagi, K., \& Tsunekawa, S. 2001, J. Mol. Spectrosc., 210, 196

Koyabashi, K., Ogata, K., Tsunekawa, S., \& Takano, S. 2007, ApJ, 657, L17

Lis, D. C., Roueff, E., Gerin, M., et al. 2002, ApJ, 571, L55

Margulès, L., Coudert, L. H., Møllendal, H., et al. 2009, J. Mol. Spectrosc., 254, 55

Margulès, L., Huet, T. R., Demaison, J., et al. 2010, ApJ, 714, 1120

Møllendal, H., Leonov, A., \& de Meijere, A. 2005, J. Phys. Chem. A, 109, 6344

Møllendal, H., Cole, G. C., \& Guillemin, J.-C. 2006, J. Phys. Chem. A, 109, 921

Motiyenko, R. A., Margulès, L., Alekseev, E. A., Guillemin, J.-C., \& Demaison, J. 2010, J. Mol. Spectrosc., 264, 94

Nummelin, A., Bergman, P., Hjalmarson, A., et al. 2000, ApJS, 128, 213

Oesterling, L. C., Albert, S., De Lucia, F. C., Sastry, K. V. L. N., \& Herbst, E. 1999, ApJ, 521, 255

Ogata, K., Odashima, H., Takagi, K., \& Tsunekawa, S. 2004, J. Mol. Spectrosc., 225, 14

Pickett, H. M., Poynter, R. L., Cohen, E. A., et al. 1998, J. Quant. Spectrosc. Radiat. Transf., 60, 883

Plummer, G. M., Herbst, E., De Lucia, F. C., \& Blake, G. A. 1984, ApJS, 55, 633

Plummer, G. M., Herbst, E., De Lucia, F. C., \& Blake, G. A. 1986, ApJS, 60, 949

Robinson, G. W., \& Cornwell, C. D. 1953, J. Chem. Phys., 21, 1436

Roueff, E., Tiné, S., Coudert, L. H., et al. 2000, A\&A, 354, L63

Saito, S., Ozeki, H., Ohishi, M., \& Yamamoto, S. 2000, ApJ, 535, 227

Shah, R. Y., \& Wootten, A. 2001, ApJ, 554, 933

Tercero, B., Margulès, L., Carvajal, M., et al. 2011, A\&A, 538, A119

Tudorie, M., Coudert, L. H., Huet, T. R., Jegouso, D., \& Sedes, G. 2011, J. Chem. Phys., 134, 074314

van der Tak, F. F. S., Schilke, P., Müller, H. S. P., et al. 2002, A\&A, 388, L53 